\title{
Using SDO's AIA to investigate energy transport from a flare's energy release site to the chromosphere ${ }^{\star}$
}

\author{
J. W. Brosius ${ }^{1}$ and G. D. Holman ${ }^{2}$ \\ 1 The Catholic University of America at NASA's Goddard Space Flight Center, Code 671, Greenbelt, MD 20771, USA \\ e-mail: Jeffrey.W.Brosius@nasa.gov \\ 2 Code 671, NASA's Goddard Space Flight Center, Greenbelt, MD 20771, USA \\ e-mail: Gordon.D.Holman@nasa.gov
}

Received 23 September 2011/ Accepted 25 November 2011

\begin{abstract}
Context. Coordinated observations of a GOES B4.8 microflare with SDO's Atmospheric Imaging Assembly (AIA) and the Ramaty High Energy Solar Spectroscopic Imager (RHESSI) on 2010 July 31 show that emission in all seven of AIA's EUV channels brightened simultaneously nearly 6 min before RHESSI or GOES detected emission from plasma at temperatures around $10 \mathrm{MK}$. Aims. To help interpret these and AIA flare observations in general, we characterized the expected temporal responses of AIA's 94, $131,171,193,211$, and $335 \AA$ channels to solar flare brightenings by combining (1) AIA's nominal temperature response functions available through SSWIDL with (2) EUV spectral line data observed in a flare loop footpoint on 2001 April 24 with the Coronal Diagnostic Spectrometer (CDS) on timescales comparable to AIA's image cadence.

Methods. The nine emission lines observed by CDS cover a wide range of formation temperature from about 0.05 to $8 \mathrm{MK}$. Line brightenings observed early during the CDS flare occurred at temperatures less than about $0.7 \mathrm{MK}$, with the largest values around $0.1 \mathrm{MK}$. These brightenings were consistent with the flare's energy transport being dominated by nonthermal particle beams. Because all of AIA's EUV channels are sensitive to emission from plasma in the 0.1 to 0.7 MK temperature range, we show that all of AIA's EUV channels will brighten simultaneously during flares like this, in which energy transport is dominated by nonthermal particle beams.

Results. The 2010 July 31 flare observed by AIA and RHESSI displays this behavior, so we conclude that such beams likely dominated the flare's energy transport early during the event. When thermal conduction from a reconnection-heated, hot $(\sim 10 \mathrm{MK})$ plasma dominates the energy transport, the AIA channels that are sensitive to emission from such temperatures (particularly the 94 and $131 \AA$ channels) will brighten earlier than the channels that are not sensitive to such temperatures (171 and $211 \AA$ ).

Conclusions. Thus, based on the differences expected between AIA's response to flares whose energy transport is dominated by nonthermal particle beams from those whose energy transport is dominated by thermal conduction, AIA can be used to determine the dominant energy transport mechanism for any given event.
\end{abstract}

Key words. Sun: activity - Sun: corona - Sun: flares - Sun: transition region - Sun: UV radiation - Sun: X-rays, gamma rays

\section{Introduction}

In the widely accepted "standard" solar flare model, pre-flare energy is stored in non-potential magnetic fields. This energy is released by magnetic reconnection, during which nonthermal particle beams are accelerated and the plasma near the reconnection site (typically thought to be in the corona) is heated. The released energy is subsequently transported to and deposited in the chromosphere either by nonthermal beam particles or by thermal conduction from the directly-heated coronal source. When the flux of energy into the chromosphere exceeds what can be shed by radiative losses, the chromosphere heats and expands. Because the density in the overlying corona is less than that in the chromosphere and photosphere, expansion of the heated chromospheric material produces larger upward than downward velocities. This process, now known as "chromospheric evaporation" (see Antonucci et al. 1999 and Bornmann 1999 for reviews) was first described by Neupert (1968). Upward velocities around $100 \mathrm{~km} \mathrm{~s}^{-1}$ or more in emission lines formed at temperatures $T \gtrsim 10 \mathrm{MK}$ during flares have long been interpreted as

* Movie is available in electronic form at http://www . aanda.org evidence for this phenomenon (Antonucci et al. 1982; Antonucci \& Dennis 1983; Zarro et al. 1988; Fludra et al. 1989; Canfield et al. 1990; Doschek 1990; Mariska et al. 1993; Mariska 1994; Silva et al. 1997; Brosius 2003, 2009; Brosius \& Phillips 2004; Milligan \& Dennis 2009). The evaporated material fills magnetic loops with hot, dense, plasma that eventually cools and fades as the flare decays.

Investigations of solar flares based on rapid cadence (7.2 to $9.8 \mathrm{~s}$ ) stare spectra obtained with the Coronal Diagnostic Spectrometer (CDS; Harrison et al. 1995) aboard the Solar and Heliospheric Observatory (SOHO) spacecraft showed that intensities of emission lines formed in plasma at transition region temperatures (e.g., the $\mathrm{O} V$ line at $629.7 \AA$, formed at $T \approx 0.25 \mathrm{MK}$ ) began to increase several minutes earlier than intensities of emission lines formed in plasma at flare temperatures (e.g., the Fe XIX line at $592.2 \AA, T \approx 8 \mathrm{MK}$; Brosius \& Phillips 2004; Brosius 2009; Brosius \& Holman 2009, 2010). Statistically significant relative Doppler velocities also typically accompany the intensity increases in the emission lines. This time difference is consistent with the chromosphere being heated predominantly by beams of nonthermal particles; if 
the chromosphere were heated predominantly by thermal conduction from a directly-heated $T \sim 10 \mathrm{MK}$ source, that source would have appeared first, before the chromosphere had a chance to respond to thermal conduction from it. When possible, CDS stare spectra were combined with X-ray observations from the Ramaty High Energy Solar Spectroscopic Imager (RHESSI; Lin et al. 2002; Hurford et al. 2002; Smith et al. 2002). RHESSI combines full disk solar imaging, wide energy coverage (3 to $17000 \mathrm{keV}$ ), and high sensitivity for investigating both nonthermal and high temperature $(\gtrsim 10 \mathrm{MK})$ thermal emission from flares and microflares.

A shortcoming of the rapid cadence CDS stare studies is their comparatively limited field of view. In previous investigations, this was partly compensated by acquiring full disk $195 \AA$ images at 12 min cadence from the Extreme-ultraviolet Imaging Telescope (EIT; Delaboudinière et al. 1995) aboard SOHO. Nevertheless, there remained some uncertainty as to what was going on outside the CDS field of view. This spatial uncertainty can be eliminated by using the Atmospheric Imaging Assembly (AIA; Lemen et al. 2011) aboard the Solar Dynamics Observatory (SDO) satellite: since AIA obtains snapshots of the entire Sun in each of its EUV channels every $12 \mathrm{~s}$, the start of any given flare or microflare is never hidden outside AIA's field of view, and can always be determined for each channel within the instrumental cadence of $12 \mathrm{~s}$.

Launched on 2010 February 11, SDO observes the Sun nearly continuously in an inclined geosynchronous orbit from which its extremely high data rate is enabled through contact with a single dedicated ground station. AIA contains seven EUV channels roughly centered on the following wavelengths, designed to include solar radiation in and around strong emission lines from the following ions: $304 \AA$ (includes emission from He II, formed at $T \approx 0.05 \mathrm{MK}), 171 \AA$ (Fe IX at $T \approx 0.63 \mathrm{MK})$, $211 \AA$ (Fe XIV at $T \approx 1.9 \mathrm{MK}), 335 \AA$ (Fe XVI at $T \approx 2.7 \mathrm{MK})$, $94 \AA$ (Fe XVIII at $T \approx 6.3 \mathrm{MK}$ ), $193 \AA$ (Fe XII at $T \approx 1.4 \mathrm{MK}$ and Fe XXIV at $T \approx 19 \mathrm{MK}$ ), and $131 \AA$ (Fe VIII at $T \approx$ $0.37 \mathrm{MK}, \mathrm{Fe} \mathrm{XX}$ at $T \approx 9.1 \mathrm{MK}, \mathrm{Fe} \mathrm{XXI}$ at $T \approx 10 \mathrm{MK}$, and Fe XXIII at $T \approx 14 \mathrm{MK}$ ). Wavelength response functions for each channel were measured in the laboratory prior to SDO's launch (Boerner et al. 2011), and are available through SSWIDL. Temperature response functions for each channel were derived (Boerner et al. 2011) by combining the measured wavelength response functions with emission line data from the CHIANTI database (Dere et al. 1997, 2009), and are also available through SSWIDL. Based on AIA's powerful combination of continuous, rapid cadence (12 s), full disk EUV imaging and wide range of temperature sensitivity, its observations are expected to provide new information on solar activity including the temperature dependence of flare start times. The use of timing differences among various EUV channels to assess the roles of thermal conduction and nonthermal particle beams in transporting the energy that drives chromospheric evaporation is one of AIA's objectives (concept study report; see http://aia.lmsal.com/ public/CSR.htm).

Here we present coordinated AIA and RHESSI observations of a GOES B4.8 microflare on 2010 July 31. AIA light curves averaged over selected areas within the active region are expected to reveal whether timing differences exist among the various channels and, hence, the importance of thermal conduction relative to nonthermal particles in transporting the energy that drives chromospheric evaporation. To help with the interpretation of these and additional AIA flare observations, we characterize the expected response of AIA's EUV channels to the onset of solar flare brightenings by combining (1) AIA's temperature response functions (Boerner et al. 2011) with (2) existing EUV spectral line flare data obtained with CDS on timescales comparable to AIA's image cadence (Brosius \& Phillips 2004). In Sect. 2 we describe the 2010 July 31 flare observational results, in Sect. 3 we present the expected temporal response of AIA's EUV channels based on CDS flare spectra obtained on 2001 April 24, in Sect. 4 we discuss implications of this work, and in Sect. 5 we summarize our findings.

\section{Observation and analysis of microflare on $\mathbf{2 0 1 0}$ July 31}

We observed NOAA Active Region 11092 on 2010 July 31 with AIA from 05:00 to 05:45 UT and with RHESSI from 05:00 to 05:40 UT, during which the GOES satellite observed a B4.8 microflare from 05:17 to 05:36 UT. We used AIA's online "cutout service" (which provides Level 1.5 data; see Lemen et al. 2011) to extract high spatial resolution ( 0.6 pixels), rapid cadence (12 s), coaligned 2.5 $\times 2.5$ images centered on $\left(-6466^{\prime \prime} 3,+113^{\prime \prime} 4\right)$ from all seven of AIA's EUV channels as well as its $1600 \AA$ channel (at 24 s cadence).

Figure 1 displays a sample of 2.5 $\times 2.5$ images of AR 11092 in four different channels observed by AIA on 2010 July 31 . The source areas A and B in which two successive microflare bursts originate are separated from each other by $30^{\prime \prime}$ or more but both connected to the sunspot toward the center of the field of view. Movies in all eight of the AIA channels (one movie showing two channels is available online) indicate that the microflare started within the area outlined by the lower dashed rectangle (A), and occurred later within the area outlined by the upper dashed rectangle (B). It is possible that the B4.8 microflare observed by GOES was actually a pair of closely spaced, closely timed sympathetic microflares.

Figure 2 displays a pair of RHESSI $3-25 \mathrm{keV}$ images that show the two spatially separated sources of brightenings that are seen in the AIA images of the microflare. The time interval for each image is indicated in the figure captions. We integrated over 1-minute intervals because the count rates were low. These images were obtained using the CLEAN technique and RHESSI front detectors 1-9, giving a spatial resolution $\sim 3^{\prime \prime}$.

Figures 3 and 4 both show 3-12 and 12-25 keV RHESSI light curves. Unfortunately, a particle event in Earth's magnetosphere contaminated these with gradual humps that peak toward the center of RHESSI's observing window, so while the resulting increased background is not expected to significantly alter the start times or peak times of the flare bursts, it effectively hides any early gradual increase to which RHESSI may have been sensitive. Note the mound in the $12-25 \mathrm{keV}$ emission atop which bursts starting at 05:17 and 05:27 UT are seen in Figs. 3 and 4. GOES, however, is unaffected by this, and shows a flat background until the start of its first recorded burst at 05:17:32 UT.

Figure 3 displays light curves for all seven of AIA's EUV wavelength channels, plus that of $1600 \AA$, averaged over area A in Fig. 1 to ensure that the microflare's starting position is included in the light curves. Solid vertical lines indicate the start of an increase in EUV emission observed by AIA no later than 05:11:34 UT, a peak in the early (precursor?) brightening at 05:13:30 UT, the start of the first impulsive rise of AIA emission at $05: 16: 44 \mathrm{UT}$, and the start of the first burst recorded by GOES at 05:17:32 UT. The first impulsive peak recorded by AIA occurred at 05:18:30 UT (simultaneously for all wavebands, within the uncertainty of the cadence), and the rise toward a secondary 
J. W. Brosius and G. D. Holman: Using AIA to study flare energy transport
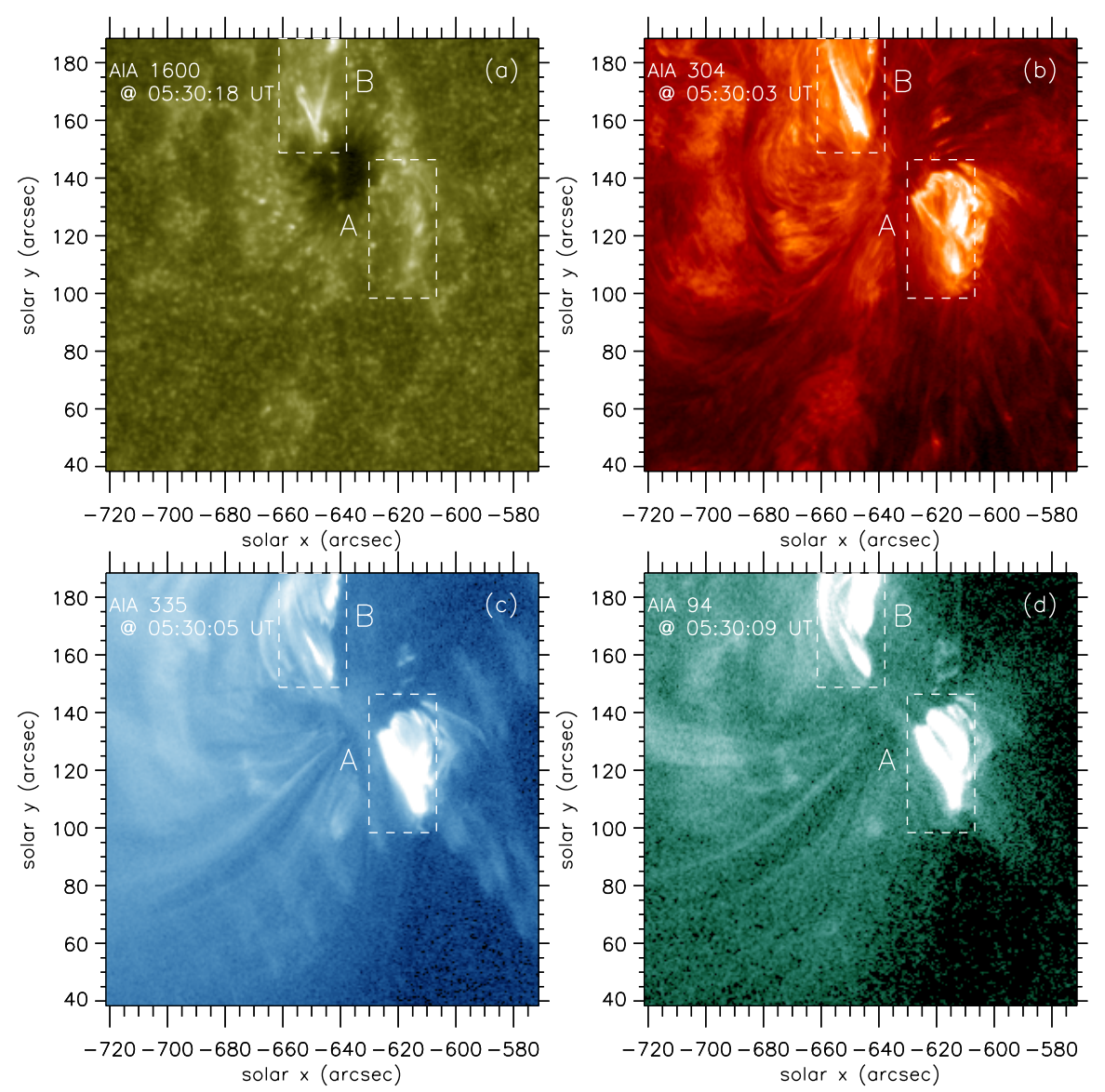

Fig. 1. $2.5 \times 2.5$ AIA images of AR 11092 in a) the $1600 \AA$ channel at 05:30:18 UT, b) the $304 \AA$ channel at 05:30:03 UT, c) the $335 \AA$ channel at 05:30:05 UT, and d) the $94 \AA$ channel at 05:30:09 UT on 2010 July 31. The dashed rectangles labeled "A" and "B" outline areas within which successive bursts were observed with AIA (first in A, later in $\mathrm{B}$ ) and RHESSI during the GOES B4.8 microflare, and for which we derived the spatially averaged AIA light curves shown in Figs. 3 and 4. Temporal evolution in the 304 and 94 A channels can be seen in the movie available online.
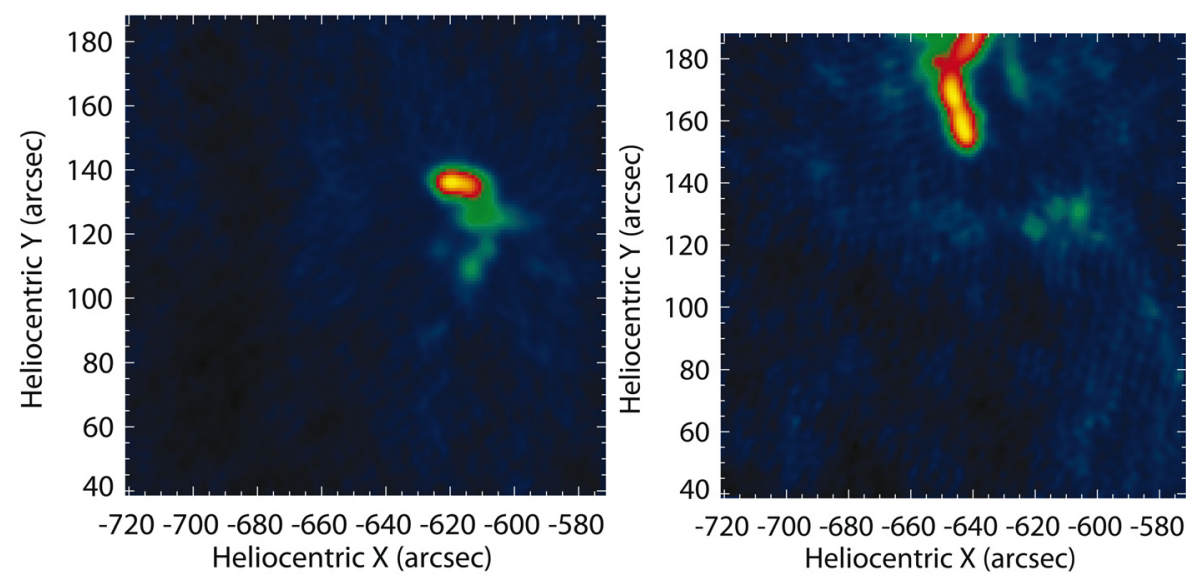

Fig. 2. 2'.5 × 2'.5 RHESSI $3-25 \mathrm{keV}$ images of the GOES B4.8 microflare integrated over 1-minute time intervals starting at 05:17:40 UT (left panel), and at 05:27:20 UT (right panel) on 2010 July 31.

peak began at 05:19:34 UT. Figure 4 displays light curves for the same AIA wavelength channels averaged over area B in Fig. 1. The GOES and RHESSI light curves from the start of the microflare component observed in area B are not as pristine as those from the start of the microflare component observed in area $\mathrm{A}$ because they are superimposed on declining emission from A, but they show that the rise in the AIA light curves at that location begins before the start of the second burst in the GOES or RHESSI light curves. Solid vertical lines indicate the start of an increase in EUV emission no later than 05:23:45 UT in $\mathrm{B}$, the start of a rapid rise in EUV emission no later than 05:26:15 UT, and the start of the second burst recorded by GOES at 05:27:08 UT.

Eventually we see different temporal behaviors in the different channels. For example, post-impulsive maxima occur at progressively later times in channels that were designed to observe bright lines of successively cooler ions, consistent with expectations of a cooling plasma after the cessation of energy deposition. During this post-impulsive cooling phase, the AIA channel that observes Fe XXI flare emission (131 A) peaks first (a somewhat broad peak centered around 05:23 UT), followed by $94 \AA$ $(05: 25 \mathrm{UT})$, and so on down to $171 \AA(05: 36 \mathrm{UT})$. The fact that the $193 \AA$ channel does not show a similar post-impulsive peak prior to that of the $131 \AA$ emission indicates that the maximum flare temperature remained smaller than that required to produce Fe XXIV.

\section{Expected temporal response of AIA based on CDS flare spectra from 2001 April 24}

To quantify the rapid cadence ( $9.8 \mathrm{~s}$ ) temporal behavior of the solar flare atmosphere over a wide range of temperature 


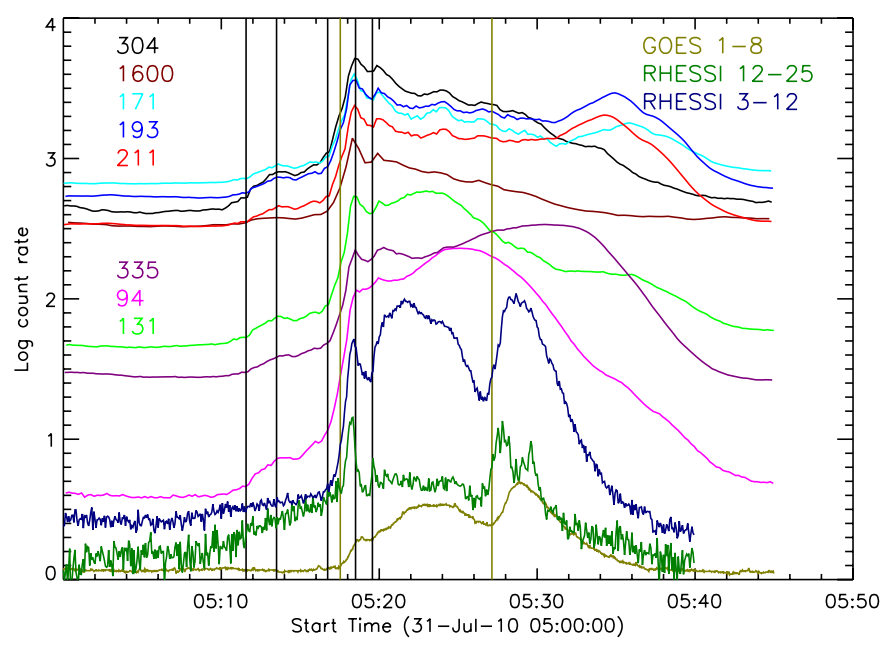

Fig. 3. Light curves (log of the count rate for AIA, arbitrary units for GOES, log of the count rate for RHESSI, but shifted downward by 2.2 for the 3-12 keV light curve, and 1.65 for the $12-25 \mathrm{keV}$ light curve) from eight wavelength channels on AIA averaged over area A (see Fig. 1) in which the brightening first occurred during the B4.8 microflare on July 31 . Solid vertical lines indicate the start of an increase in EUV emission observed by AIA no later than 05:11:34 UT, a peak in the early (precursor?) brightening at 05:13:30 UT, the start of an impulsive rise in AIA emission at 05:16:44 UT, the start of the first burst recorded by GOES at 05:17:32 UT, the first impulsive peak detected by AIA at 05:18:30 UT (simultaneously for all wavebands, within the uncertainty of the cadence), the rise toward a secondary peak at 05:19:34 UT, and the start of the second burst recorded by GOES at 05:27:08 UT.

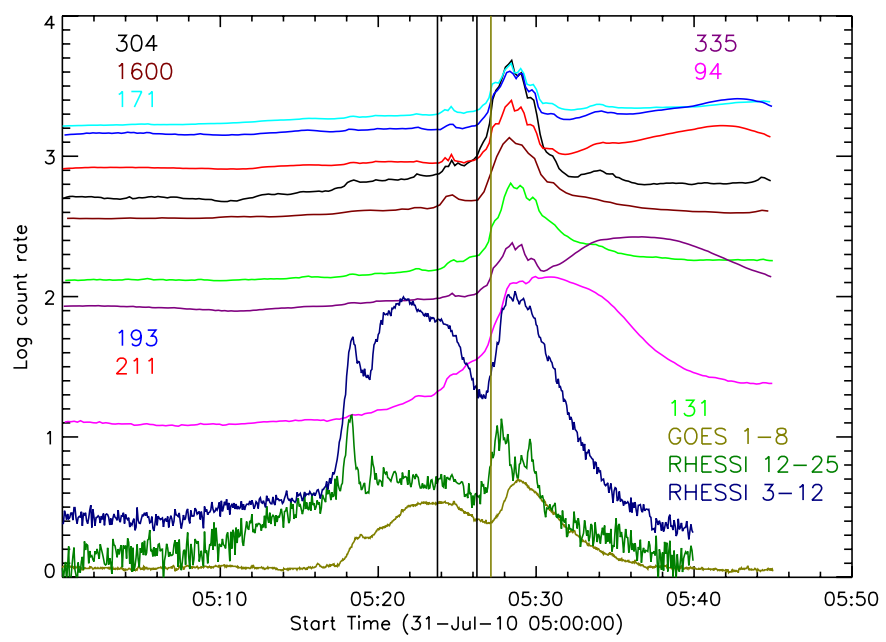

Fig. 4. Light curves (log of the count rate for AIA, arbitrary units for GOES, log of the count rate for RHESSI, but shifted downward by 2.2 for the 3-12 keV light curve, and 1.65 for the $12-25 \mathrm{keV}$ light curve) from eight wavelength channels on AIA averaged over area B (see Fig. 1) in which the brightening started later during the B4.8 microflare on July 31 . Solid vertical lines indicate the start of an increase in EUV emission observed by AIA no later than 05:23:45 UT, the start of a rapid rise in EUV emission no later than 05:26:15 UT, and the start of the second burst recorded by GOES at 05:27:08 UT.

(4.67 $\leq \log T \leq 6.89$ ), we use the integrated intensities (ergs $\mathrm{cm}^{-2} \mathrm{~s}^{-1} \mathrm{sr}^{-1}$ ) derived by Brosius \& Phillips (2004) for the following nine emission lines measured at the commencement site (an area in and around one of the flare loop's footpoints) of a GOES M2.3 flare in Active Region 9433 on 2001 April 24: He II $303.78 \AA$ ( $\log T=4.67)$, O III 599.60 $(\log T=4.96)$,

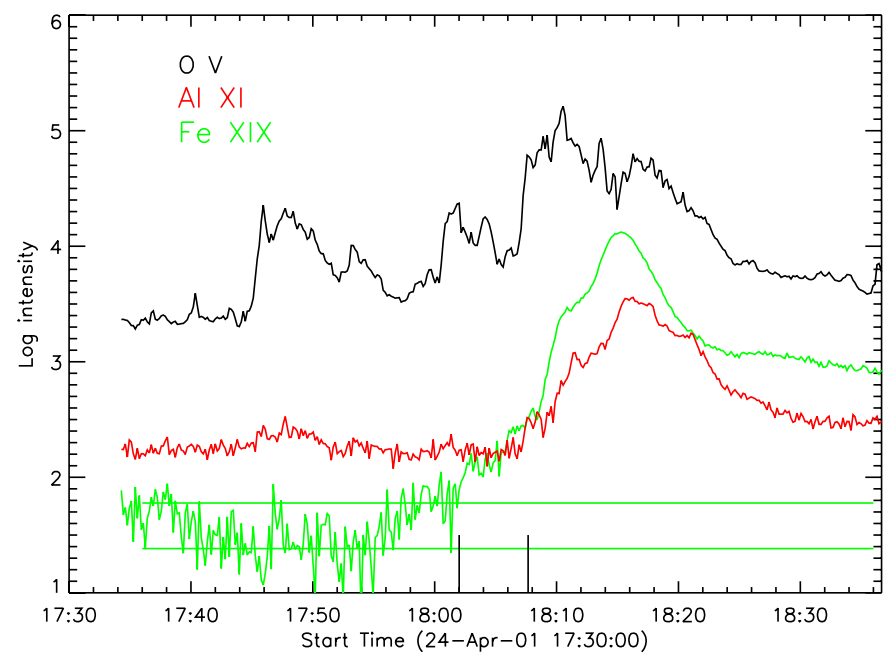

Fig. 5. Light curves of O V $629.7 \AA$, Al XI 550.0 ̊, and Fe XIX 592.2 ̊ obtained with SOHO's CDS in rapid cadence $(9.8 \mathrm{~s})$ stare mode during the 2001 April 24 GOES M2.3 flare reported by Brosius \& Phillips (2004). We use these and light curves from six additional spectral lines to measure average preflare intensities and flare intensity enhancement factors as described in the text. Solid horizontal lines indicate $\pm 1 \sigma$ from the average pre-flare Fe XIX noise. The long vertical tick mark at 18:02:00 UT indicates the time at which the Fe XIX intensity exceeds and remains continuously greater than its pre-flare noise level by more than $1 \sigma$, and the long vertical tick mark at 18:07:40 UT indicates the time at which Yohkoh hard X-ray count rates became statistically significant (with a peak at 18:10:40 UT).

O IV $554 \AA$ (a self-blend of four lines at 553.33, 554.08, 554.51, and 555.26 $\AA$, formed at $\log T=5.24), \mathrm{O}$ V $629.76 \AA$ $(\log T=5.37), \mathrm{Ne}$ VI $558.59 \AA(\log T=5.63), \mathrm{Ca} X 557.76 \AA$ ( $\log T=5.87), \operatorname{Mg}$ X $609.79 \AA$ ( $\log T=6.05$, from which its $\mathrm{O}$ IV blend was removed based on the density-insensitive intensity ratio O IV $609.83 /(553.33+554.08+554.51+555.26)=$ $0.1645 \pm 0.0022$ derived with CHIANTI v. 6.0.1; Dere et al. 1997, 2009), Al XI 550.04 $(\log T=6.17)$, and Fe XIX $592.23 \AA(\log T=6.89)$. See Fig. 5. Using the 51 exposures whose start times occurred between 17:36:05 and 17:44:18 UT, we derived pre-flare average intensities and their corresponding 1-sigma scatters. All of the lines except that of Fe XIX at 592.2 A were observed during this pre-flare interval; for Fe XIX the pre-flare average intensity is defined by the noise. We consider the detection of Fe XIX emission to be reliable only when the intensity derived from fits to the line profile exceeds and continuously remains greater than the pre-flare average noise level by more than $1 \sigma$ (which occurs at 18:02:00 UT; see Fig. 5). Upward velocities approaching $-40 \mathrm{~km} \mathrm{~s}^{-1}$ in only the transition region lines of $\mathrm{O}$ III, O IV, O V, and He II during the "precursors" (17:44:30-17:57:10 and 18:00:30-18:05:30 UT) indicated gentle chromospheric evaporation; downward velocities of +40 $\mathrm{km} \mathrm{s}^{-1}$ in those same lines during the impulsive phase, when Fe XIX showed maximum upward velocities of $-64 \mathrm{~km} \mathrm{~s}^{-1}$, indicated explosive evaporation.

For each exposure beginning with the start of the first precursor, we calculate an intensity enhancement factor for each emission line as the ratio of the line's intensity in the given exposure to its pre-flare average value. Since the Fe XIX line emission is considered to be noise until its intensity continuously exceeds the pre-flare average noise by more than $1 \sigma$, we define its enhancement factor as 1 until that time. Thus for each $9.8 \mathrm{~s}$ interval we calculate 9 enhancement factors, and we 
assume that each corresponds to its respective emission line's formation temperature (listed above). We use a spline fit to place these onto the finer temperature grid $(0.05 \mathrm{dex})$ of the AIA temperature response functions (Boerner et al. 2011). In this way we obtain enhancement factors as functions of temperature for $4.65 \leq \log T \leq 6.90$. See Fig. 6, which shows normalized AIA temperature response functions for all of the EUV channels except $304 \AA$ A along with normalized enhancement factor curves for six CDS exposures up to and including the first peak during the flare's first precursor. In Fig. 6 and subsequent figures, the AIA temperature response functions are normalized to the maximum value within their respective channels. The EUV spectral line enhancement factors are normalized to the largest overall value during the flare (325, which occurs for Fe XIX during its peak at 18:15:18 UT), multiplied by 10 in Fig. 6 to improve visibility. The point of Fig. 6 is that the majority of the intensity enhancements observed early during the first precursor occur at temperatures less than about $\log T=5.8(0.6 \mathrm{MK})$, with the largest values occurring around $\log T=5.0(0.1 \mathrm{MK})$.

Figure 7 is similar to Fig. 6, and shows normalized enhancement factors (in this case not multiplied by a factor of 10) for four CDS exposures during the flare. These include the first peak during the first precursor (17:45:56 UT, which is also shown in Fig. 6), the start of the impulsive increase of the Fe XIX emission at 18:08:24 UT, the peak intensity of the cool, transition region emission lines at 18:10:32 UT, and the peak intensity of the Fe XIX line observed by CDS at 18:15:18 UT (the maximum to which all of the CDS enhancements are normalized). It is evident that although intensity enhancements due to low temperature emission dominate early during the flare, by the time of the maximum $\mathrm{O} \mathrm{V}$ intensity the contributions from hot flare emission (approaching $10 \mathrm{MK}$ ) are significant while at the time of the maximum Fe XIX intensity they dominate the low temperature contributions.

Figure 8 shows fractional contributions to the total brightness increase in AIA's 94, 131, 171, 193, 211, and $335 \AA$ channels due to plasma with $\log T \leq 5.85$. Because the temperature range for this calculation is limited to that available to CDS (4.67 $\leq \log T \leq 6.89$ ), we define the fractional contribution for each frame in this figure as the ratio of the enhancement summed over the interval from $\log T=4.65$ to 5.85 to that summed over the interval from $\log T=4.65$ to 6.90 . The curves show that contributions to the total brightness increase in each channel are greatest for low-temperature emission until about 18:10 UT, when contributions from emission formed at larger temperatures begin to dominate. The lower, darker curve in Figure 8a was calculated with the $94 \AA$ channel's nominal temperature response function (Boerner et al. 2011) obtained through SSWIDL, while the upper, lighter curve was calculated with a "corrected" version of this function in which the temperature response was multiplied by 5 for $\log T \leq 5.85$ (see discussion).

Based on our combination of the nominal AIA temperature response curves available through SSWIDL (Boerner et al. 2011) with the (temperature-dependent) intensity enhancement factors derived from the CDS spectral line data of the M2.3 flare on 2001 April 24 (Brosius \& Phillips 2004), we estimate that AIA would have observed count rate increases in its $94 \AA$ channel by factors of 1.87 at the first peak of the flare's first precursor, 6.94 by the start of the impulsive rise of the Fe XIX emission, 40.6 at the overall maximum in the "cool" (e.g., O V) line emission, and 186.5 at the time of the overall maximum in the Fe XIX flare line emission. These and corresponding values at the same times in the $131,171,193,211$, and $335 \AA$ channels are listed in Table 1. By way of comparison, AIA channel enhancement factors observed on 2010 July 31 at the peak (05:13:30 UT) of the earliest flare (precursor?) brightening and the first impulsive peak (05:18:30 UT) of the light curves from area A (see Fig. 1) are given in Table 1.

All of AIA's EUV channels are sensitive to emission around $\log T \approx 5.5$ (see Figs. 6 and 7). This means that any flare (like that observed by Brosius \& Phillips 2004) in which emission from plasma at $\log T \approx 5.5$ brightens significantly earlier (by more than AIA's $12 \mathrm{~s}$ cadence) than emission from plasma at "flare" temperatures $(\log T \approx 7)$ will brighten simultaneously in AIA's 94, 131, 171, 193, 211, and 335 (and 304) ^ channels. As can be seen from AIA's temperature response functions shown in Figs. 6 and 7, all of those channels are also sensitive to emission from plasma at $\log T \approx 6$. Thus, while a simultaneous brightening in all of AIA's EUV channels would be consistent with previous spectroscopic observations in which emission from plasma at $\log T \approx 5.5$ is the first to brighten during a flare (e.g., Brosius \& Phillips 2004; Brosius 2009; Brosius \& Holman $2009,2010)$, the possibility would remain that the actual temperature of the emitting plasma is close to $\log T \approx 6$ or perhaps spread over a range from $\log T \approx 5$ to $\log T \approx 6$. Thus for AIA flare observations in which all of AIA's EUV channels brighten simultaneously we encounter some ambiguity regarding the temperature distribution of the emitting plasma. Nevertheless, a simultaneous brightening in all of AIA's EUV channels is consistent with chromospheric heating and evaporation being driven by beams of nonthermal particles.

If, on the other hand, emission from plasma at $\log T \approx 7$ (the result of direct coronal heating at the reconnection site) brightened significantly before emission from plasma at $\log T \approx 5.5$, then only the AIA channels that are sensitive to emission from such hot plasma (94 and $131 \AA$ ) will brighten significantly while others (193 and $335 \AA$ ) may show a smaller brightening and the remainder (171 and $211 \AA$ ) will show no effect. This enables AIA to distinguish which mechanism (thermal conduction or nonthermal particle beam) dominates the transport of flare energy from its release site (typically thought to be in the corona) to the chromosphere. By way of quantifying AIA's flare response for a case in which reconnection-heated, hightemperature $(10 \mathrm{MK})$ plasma appears before the brightening of plasma at lower temperatures, we consider a synthetic case in which only the intensity of the hot flare plasma $(6.8 \leq \log$ $T \leq 6.9)$ is enhanced early during the flare. Based on calculations similar to those described above for the combination of AIA temperature response curves and CDS flare spectra, we find that the $94 \AA$ channel would brighten by a factor of 4.51 when the intensity enhancement factor is 10 , and by a factor of 12.3 when it is 30. Similarly, the $131 \AA$ channel would brighten by factors of 1.18 and 1.57 for the same intensity enhancement factors. For the $171 \AA$ channel we get 1.00 and 1.00 , for the $193 \AA$ channel we get 1.03 and 1.09 , for the $211 \AA$ channel we get 1.02 and 1.08, and for the $335 \AA$ channel we get 1.18 and 1.57 .

\section{Discussion}

Figure 3 shows that all of the AIA light curves from area A in Fig. 1 started to rise at the same time, nearly 6 min before RHESSI or GOES detected any increase due to the microflare, while Fig. 4 shows that all of the AIA light curves from area B started their rise more than 3 min before RHESSI or GOES detected an increase. This is consistent with expectations presented in Sect. 3, in which the simultaneous increase observed in AIA's 

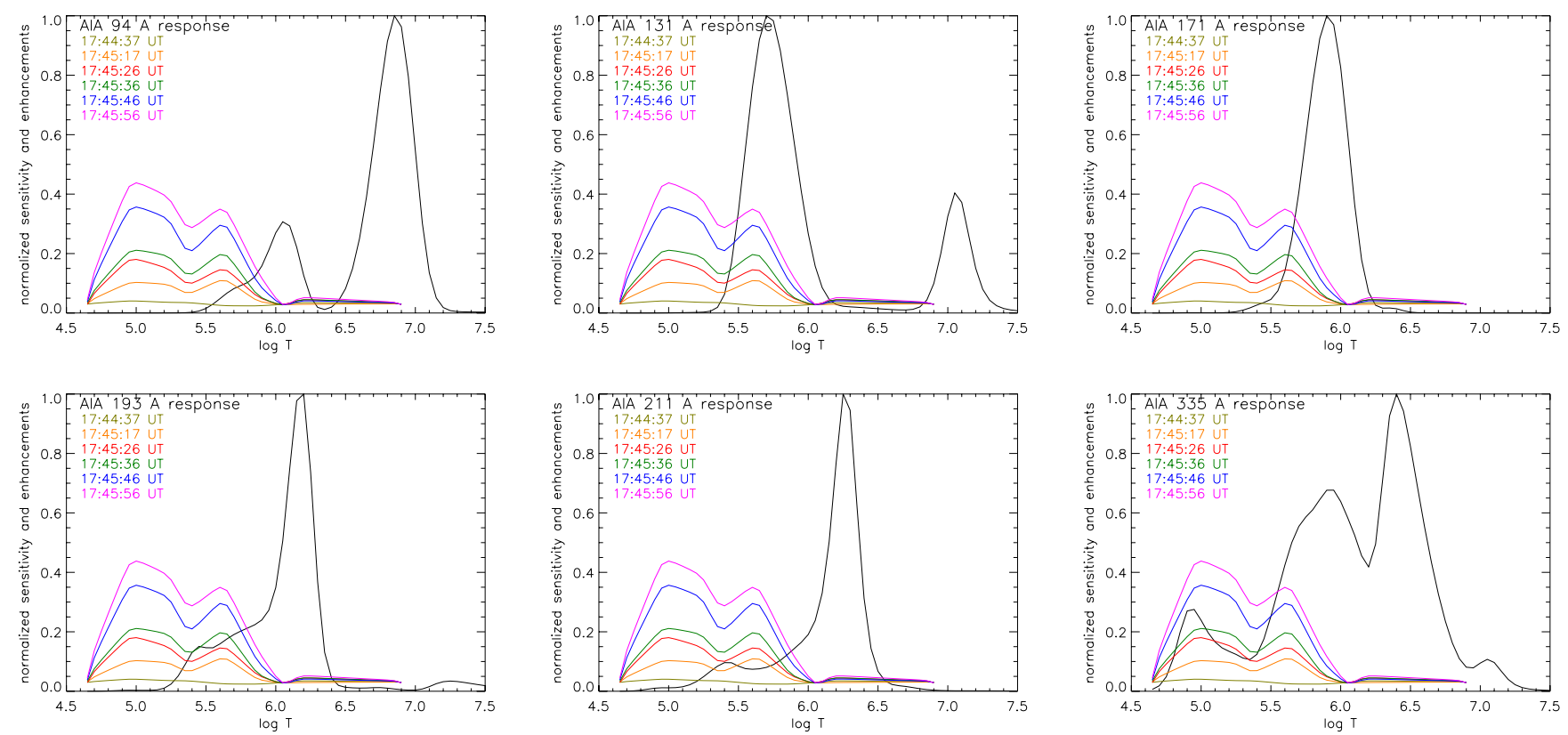

Fig. 6. Nominal, normalized AIA temperature response functions (Boerner et al. 2011), with CDS enhancement factors (normalized to the largest value which occurs for Fe XIX, then multiplied by 10 to improve visibility in these frames) for six of the first ten CDS exposures, beginning with the first exposure during the first precursor and ending with the first peak during the first precursor of the M2.3 flare. The interval between exposures is $9.8 \mathrm{~s}$. The majority of the intensity enhancements occur at temperatures less than $\log T=5.8$, with the $\operatorname{largest}$ values at $\log T=5.0$ (for the $\operatorname{last}$ four CDS exposures in this series). The greatest contributions to AIA's brightenings occur where the product of the intensity enhancements and the response functions are largest.
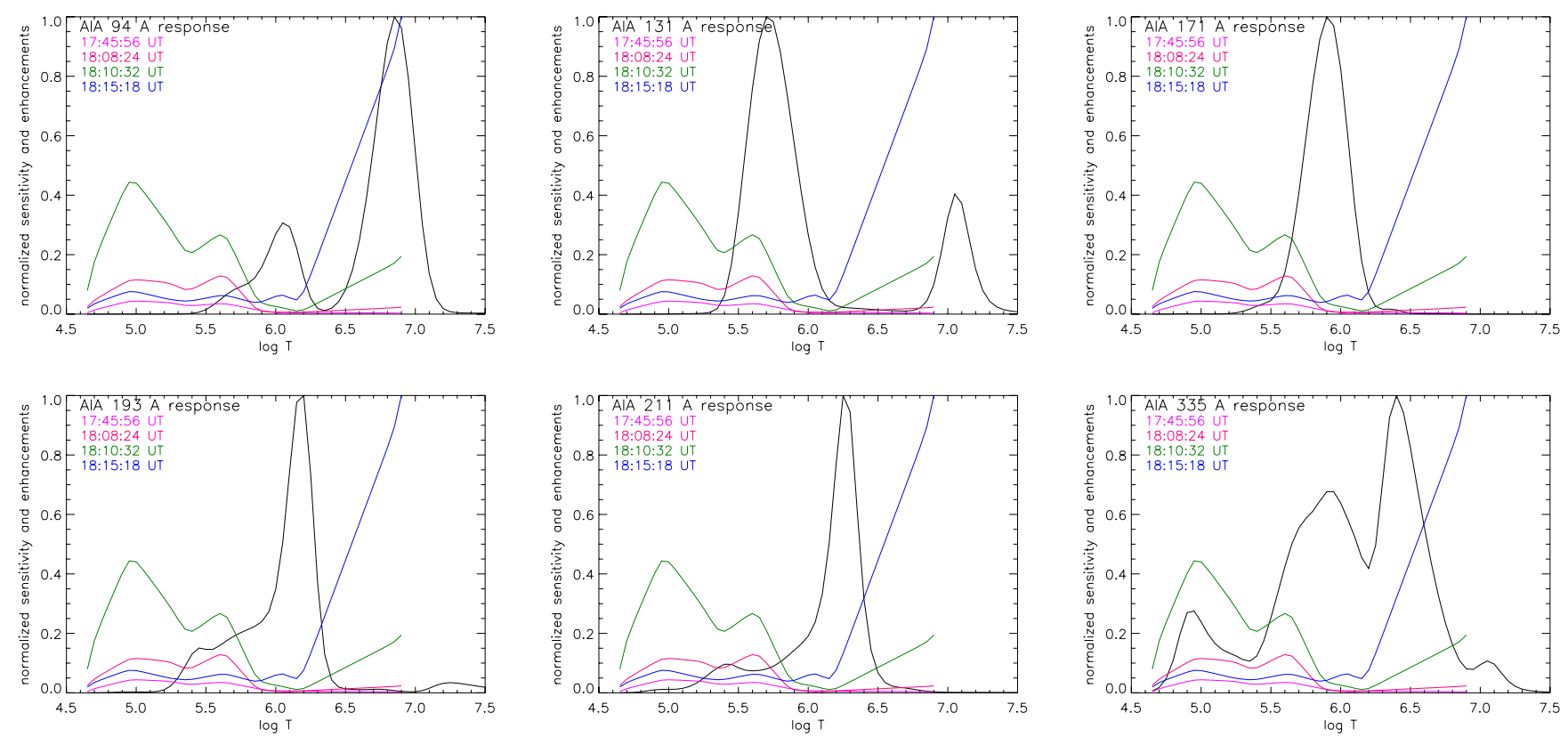

Fig. 7. Nominal, normalized AIA temperature response functions, with CDS enhancement factors (normalized to the largest value which occurs for Fe XIX) for four times during the M2.3 flare, including (1) the first peak of the first precursor at 17:45:56 UT (the top-most CDS curve in Fig. 6, but here not multiplied by 10), (2) the start of the impulsive increase of the Fe XIX emission at 18:08:24 UT, (3) the peak intensity of the cool, transition region emission lines at 18:10:32 UT, and (4) the peak intensity of the Fe XIX line observed by CDS at 18:15:18 UT (the maximum to which all of the CDS enhancements are normalized). The interval between exposures is $9.8 \mathrm{~s}$.

$94,131,171,193,211$, and $335 \AA$ channels is due to emission from plasma at temperatures between 0.1 and $0.7 \mathrm{MK}$. This is consistent with nonthermal electrons starting to heat the chromosphere nearly 6 min before RHESSI or GOES detected microflare emission. If the chromosphere were heated by thermal conduction, an earlier increase in high-temperature ( $\gtrsim 10 \mathrm{MK})$ emission is expected to have been seen by RHESSI, GOES, or the AIA channels that were designed with the greatest sensitivity to emission from high-temperature flare plasma (Fe XVIII at 6.3 MK in the $94 \AA$ channel, Fe XXI at $10 \mathrm{MK}$ in the $131 \AA$ 

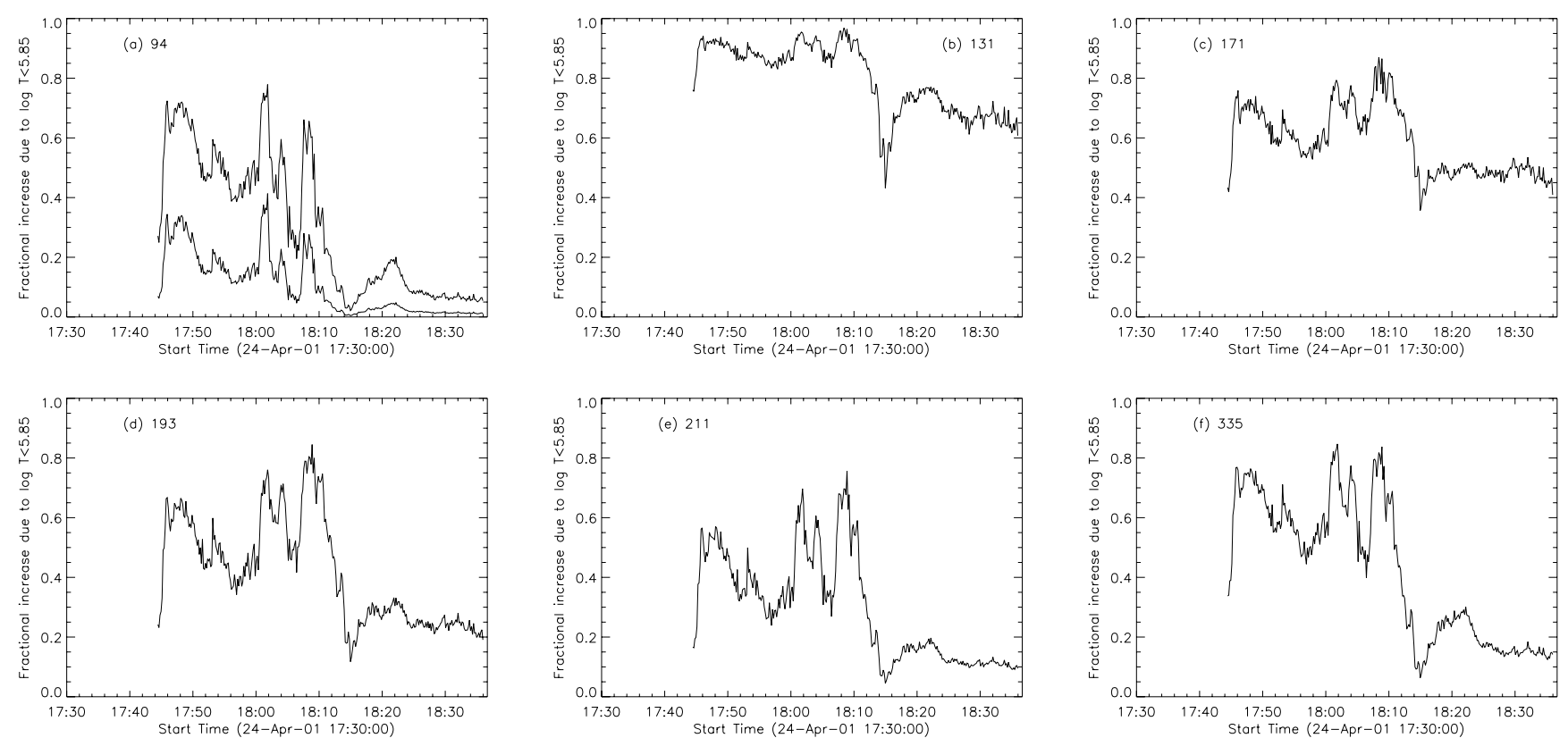

Fig. 8. Fractional contribution to total brightness increase in six AIA EUV channels attributed to plasma with $\log T \leq 5.85$. For this investigation our temperature range is constrained to that available to CDS, namely, $4.67 \leq \log T \leq 6.89$. We increased AIA's $94 \AA$ A channel's low-temperature (4.65 $\leq \log T \leq 5.85)$ sensitivity by a factor of 5 to compensate for its known underestimate of its low-temperature sensitivity; this is plotted as the upper (lighter color) curve in frame a), as described further in the text.

Table 1. AIA intensity enhancement factors at selected times during flares.

\begin{tabular}{cccccccc}
\hline \hline & \multicolumn{4}{c}{ Based on AIA and CDS $^{a}$} & & \multicolumn{2}{c}{ AIA observed $^{b}$} \\
\cline { 3 - 4 } \cline { 5 - 7 } Channel & Pre peak $^{c}$ & Rise XIX $^{d}$ & O V Max $^{e}$ & Max XIX $^{f}$ & & 1st peak $^{g}$ & Imp. peak $^{h}$ \\
\hline $94 \AA$ & 1.87 & 6.94 & 40.6 & 186.5 & 1.85 & 29.2 \\
$131 \AA$ & 7.51 & 22.9 & 51.2 & 25.0 & 1.63 & 11.5 \\
$171 \AA$ & 4.65 & 10.7 & 25.8 & 17.3 & 1.33 & 6.00 \\
$193 \AA$ & 3.58 & 9.14 & 22.2 & 27.4 & 1.38 & 6.74 \\
$211 \AA$ & 3.08 & 7.45 & 21.5 & 45.2 & 1.33 & 7.10 \\
$335 \AA$ & 4.62 & 12.7 & 40.8 & 67.9 & 1.35 & 7.85 \\
\hline
\end{tabular}

Notes. ${ }^{(a)}$ Expected AIA intensity enhancement factor based on nominal temperature response functions (Boerner et al. 2011) combined with intensity enhancement factors measured with CDS spectra during the M2.3 flare on 2001 April 24 (Brosius \& Phillips 2004). ${ }^{(b)}$ Values observed by AIA in area A of Fig. 1 during the microflare on 2010 July 31. ${ }^{(c)}$ At the first peak of the flare's first precursor (17:45:56 UT). ${ }^{(d)}$ At the impulsive rise of the Fe XIX emission (18:08:24 UT). ${ }^{(e)}$ At the maximum intensity observed in the cool (e.g., O V) emission lines (18:10:32 UT). ${ }^{(f)}$ At the maximum of the Fe XIX emission (18:15:18 UT). ${ }^{(g)}$ At the first peak after the microflare's onset (05:13:30 UT). ${ }^{(h)}$ At the microflare's first impulsive peak (05:18:30 UT).

channel, Fe XXIV at 19 MK in the $193 \AA$ channel, and Ni XXII at $10 \mathrm{MK}$ and Fe XXI at $10 \mathrm{MK}$ in the $335 \AA$ channel). Since this was not the case, we find no observational evidence to suggest that thermal conduction heated the chromosphere or drove evaporation at the start of the microflare (in either area A or area B).

The RHESSI data show no X-ray emission before about 15:16 UT. After this time, the $12-25 \mathrm{keV}$ light curve (see Fig. 3) displays the impulsive character of bremsstrahlung from nonthermal electrons. The first X-ray peak, beginning between 5:17 UT and 5:18 UT and ending between 5:19 UT and 5:20 UT, may in fact contain significant emission from nonthermal electrons down to the lowest observed photon energy of $3 \mathrm{keV}$. Spectral analysis to investigate the likelihood that the higher energy photons (and a significant fraction of the lower energy X-ray photons in the first peak) were from nonthermal electrons was inconclusive because of the low level of emission above $12 \mathrm{keV}$ and the uncertainty in the background subtraction. Despite RHESSI's observation of no hard X-ray emission before 5:16 UT, nonthermal electrons could still have provided the power input required to heat the chromospheric plasma at these earlier times (e.g., Siarkowski et al. 2009; Falewicz et al. 2011).

In addition to the light curves from areas $\mathrm{A}$ and $\mathrm{B}$, we also obtained light curves averaged over the entire $2.5 \times 2.5$ field of view that we had selected from the AIA cutout service. Because these light curves are averaged over large areas that do not participate in the flare brightenings, features in them appear relatively weakened or suppressed when compared with their counterparts from areas A and B. Although this makes the starting times of some of the features indicated in Figs. 3 and 4 more difficult to determine, the relative timings remain unchanged. Further, and 
perhaps most important, is the fact that the light curves averaged over this large area yield no evidence of a "hot" $(T \sim 10 \mathrm{MK})$ start that may have occurred outside areas A and B above. This provides further support for our conclusion that flare energy was transported by nonthermal particle beams rather than by thermal conduction during the 2010 July 31 B4.8 microflare.

In a recent theoretical investigation O'Dwyer et al. (2010) computed the contributions of spectral lines and continuum emission to AIA's EUV channels for different regions of the solar atmosphere (coronal hole, quiet Sun, active region, and flare plasma), and concluded that no waveband is strictly isothermal: emission from plasma at a variety of temperatures contributes significantly to each. Although Table 1 of O'Dwyer et al. (2010) indicates that AIA's 94, 131, and $193 \AA$ channels are dominated by Fe XVIII, Fe XXI, and Fe XXIV, respectively, during a flare, those calculations were performed with a differential emission measure obtained during the decay of an M2 flare (Dere \& Cook 1979). This DEM did not include the flare impulsive phase, when bursts of emission from plasma at transition region temperatures $(0.1-0.5 \mathrm{MK})$ are particularly bright (see, e.g., Fisher et al. 1985a,b,c; and CDS light curves presented by Brosius 2003; Brosius \& Phillips 2004 and Brosius \& Holman 2007). Thus, as we have demonstrated in Sect. 3 above, emission from transition region plasma will dominate in all of AIA's EUV channels during a flare's precursors and early impulsive rise, while emission from plasma at temperatures around $10 \mathrm{MK}$ will dominate later during the event. In an even more recent comparison of AIA images of a non-flaring loop footpoint and leg with coordinated EUV spectra from Hinode's Extreme-ultraviolet Imaging Spectrometer (EIS, Culhane et al. 2007), Del Zanna et al. (2011) showed that emission from lines formed at transition region temperatures can be significant for all of AIA's EUV channels, and dominant in some cases. Their observation of numerous unidentified lines in the EIS spectra underscored the need for further work on the relevant atomic data before AIA images can be reliably used for plasma diagnostic purposes.

A number of emission lines that are found, for example, in the Kelly (1987) Finding List at wavelengths between 93.5 and 94.5 $\AA$ are absent from the CHIANTI v.6.0.1 database (Dere et al. 1997, 2009) on which the AIA temperature response functions as of this writing are based (Boerner et al. 2011). These include 11 lines of $\mathrm{Al} \mathrm{V}(\log T=5.42), 1$ line of $\mathrm{O} \mathrm{VI}$ $(\log T=5.46), 1$ line of Na VI $(\log T=5.65), 2$ lines of Al VII $(\log T=5.79), 10$ lines of Na VII $(\log T=5.78)$, and 2 lines of Na VIII $(\log T=5.86)$. Any or all of these might significantly increase the low-temperature sensitivity of the $94 \AA$ channel's temperature response function. For demonstration purposes, we multiplied the existing, nominal $94 \AA$ channel's temperature response function (Boerner et al. 2011; available through SSWIDL, and used to derive the lower, darker curve in Fig. 8a) by a factor of 5 for $\log T \leq 5.85$. This value is at the low end of the $6.7 \pm 1.7$ range in correction factor proposed by Aschwanden \& Boerner (2011), but it is difficult to say what the correction factor really is without the additional relevant atomic physics data that need to be generated and included in the CHIANTI database. We repeated the calculation of the fractional contribution to the total brightness increase in AIA's $94 \AA$ channel due to plasma with $\log T \leq 5.85$, and display the result as the upper, lighter-color curve in Fig. 8a. Note that the greater sensitivity to low temperature emission translates to a larger fractional contribution to flare brightening due to this emission. It does not, however, alter our conclusions regarding the simultaneity of flare brightenings in AIA's EUV channels. Further, it appears likely that the $131 \AA$ channel's temperature response function will undergo a similar revision once the 7 lines of $\mathrm{O} \mathrm{V}(\log T=5.37), 4$ lines of $\mathrm{Al} \mathrm{V}(\log T=5.42), 8$ lines of $\mathrm{Na} \mathrm{V}(\log T=5.46)$, and 8 lines of $\mathrm{Ne} \mathrm{V}(\log T=5.47)$ in the Finding List between wavelengths of about 128 and $132 \AA$ are also available through CHIANTI to be included in the $131 \AA$ channel's temperature response function.

Furthur studies that compare AIA images with coordinated CDS, EIS, or EUNIS (Extreme Ultraviolet Normal Incidence Spectrograph; Brosius et al. 2008; Wang et al. 2010, 2011) spectral observations that cover AIA channels accessible to those instruments will help to better quantify the temperature responses of those channels by directly showing all of the spectral lines that produce the observed count rates in selected areas within a variety of solar features.

\section{Summary}

Coordinated AIA and RHESSI observations of a GOES B4.8 microflare on 2010 July 31 show that emission in all seven of AIA's EUV channels brightened simultaneously nearly 6 minutes before RHESSI or GOES detected emission from plasma at temperatures around $10 \mathrm{MK}$. To help interpret these and AIA flare observations in general, we characterized the expected temporal responses of AIA's 94, 131, 171, 193, 211, and $335 \AA$ channels to solar flare brightenings by combining (1) AIA's nominal temperature response functions available through SSWIDL (Boerner et al. 2011) with (2) EUV spectral line flare data obtained with CDS on 2001 April 24 (Brosius \& Phillips 2004) on timescales comparable to AIA's image cadence. The line brightenings that were observed early during the CDS flare occurred at temperatures less than about $0.7 \mathrm{MK}$, with the largest values around $0.1 \mathrm{MK}$; this is consistent with the flare's energy transport being dominated by nonthermal particle beams. Because all of AIA's EUV channels are sensitive to emission from plasma in the 0.1 to $0.7 \mathrm{MK}$ temperature range, we show that all of AIA's EUV channels will brighten simultaneously during flares like this, in which energy transport is dominated by nonthermal particle beams. The 2010 July 31 flare observed by AIA and RHESSI displays this behavior, and is consistent with the idea that particle beams transported the energy from the energy release site to the chromosphere, where it began to drive chromospheric evaporation nearly $6 \mathrm{~min}$ before flare temperatures around $10 \mathrm{MK}$ were reached. When thermal conduction from a directly-heated, hot ( $10 \mathrm{MK})$ plasma is the dominant energy transport mechanism, the AIA channels that are sensitive to emission from such temperatures (particularly the 94 and $131 \AA$ channels) will brighten earlier than the channels that are not sensitive to such temperatures (171 and $211 \AA$ ). Thus, based on the differences between AIA's response to flares whose energy transport is dominated by nonthermal particle beams from those whose energy transport is dominated by thermal conduction, AIA can be used to determine the dominant energy transport mechanism for any given event.

Acknowledgements. J.W.B. acknowledges NASA support through SR\&T grant NNX10AC08G. G.D.H. acknowledges partial support from SR\&T grant NNX10AC08G and the RHESSI Project. The AIA data used are provided courtesy of NASA/SDO and the AIA science team.

\section{References}

Antonucci, E., \& Dennis, B. R. 1983, Sol. Phys., 86, 67 Antonucci, E., Gabriel, A. H., Acton, L. W., et al. 1982, Sol. Phys., 78, 107 
J. W. Brosius and G. D. Holman: Using AIA to study flare energy transport

Antonucci, E., Alexander, D., Culhane, J. L., et al. 1999, in The Many Faces of the Sun, ed. K. T. Strong, J. L. R. Saba, B. M. Haisch, \& J. T. Schmelz (New York: Springer), 331

Aschwanden, M. J., \& Boerner, P. 2011, ApJ, 732, 81

Boerner, P., Edwards, C., Lemen, C., et al. 2011, Sol. Phys., DOI: $10.1007 / \mathrm{s} 11207-011-9804-8$

Bornmann, P. L. 1999, in The Many Faces of the Sun, ed. K. T. Strong, J. L. R. Saba, B. M. Haisch, \& J. T. Schmelz (New York: Springer), 301

Brosius, J. W. 2003, ApJ, 586, 1417

Brosius, J. W. 2009, ApJ, 701, 1209

Brosius, J. W., \& Holman, G. D. 2007, ApJ, 659, L73

Brosius, J. W., \& Holman, G. D. 2009, ApJ, 692, 492

Brosius, J. W., \& Holman, G. D. 2010, ApJ, 720, 1472

Brosius, J. W., \& Phillips, K. J. H. 2004, ApJ, 613, 580

Brosius, J. W., Rabin, D. M., Thomas, R. J., \& Landi, E. 2008, ApJ, 677, 781

Canfield, R. C., Zarro, D. M., Metcalf, T. R., \& Lemen, J. R. 1990, ApJ, 348, 333

Culhane, J. L., Harra, L. K., James, A. M., et al. 2007, Sol. Phys., 243, 19

Delaboudinière, J.-P., Artzner, G. E., Brunaud, J., et al. 1995, Sol. Phys., 162, 291

Del Zanna, G., O’Dwyer, B., \& Mason, H. E. 2011, A\&A, 535, A46

Dere, K. P., \& Cook, J. W. 1979, ApJ, 229, 772

Dere, K. P., Landi, E., Mason, H. E., Monsignori Fossi, B. C., \& Young, P. R. 1997, A\&AS, 125, 149

Dere, K. P., Landi, E., Young, P. R., et al. 2009, A\&A, 498, 915

Doschek, G. A. 1990, ApJS, 73, 117

Falewicz, R., Siarkowski, M., \& Rudawy, P. 2011, ApJ, 733, 37
Fisher, G. H., Canfield, R. C., \& McClymont, A. N. 1985a, ApJ, 289, 414 Fisher, G. H., Canfield, R. C., \& McClymont, A. N. 1985b, ApJ, 289, 425 Fisher, G. H., Canfield, R. C., \& McClymont, A. N. 1985c, ApJ, 289, 434 Fludra, A., Lemen, J. R., Jakimiec, J., Bentley, R. D., \& Sylwester, J. 1989, ApJ, 344, 991

Harrison, R. A., Sawyer, E. C., Carter, M. K., et al. 1995, Sol. Phys., 162, 233 Hurford, G. J., Schmahl, E. J., Schwartz, R. A., et al. 2002, Sol. Phys., 210, 61 Kelly, R. L. 1987, J. Phys. Chem. Ref. Data, 16 (Finding List) Lemen, J. R., Title, A. M., Akin, D. J., et al. 2011, Sol. Phys., DOI: $10.1007 / \mathrm{s} 11207-011-9776-8$

Lin, R. P., Dennis, B. R., Hurford, G. J., et al. 2002, Sol. Phys., 210, 3

Mariska, J. T. 1994, ApJ, 434, 756

Mariska, J. T., Doschek, G. A., \& Bentley, R. D. 1993, ApJ, 419, 418

Milligan, R. O., \& Dennis, B. R. 2009, ApJ, 699, 968

Neupert, W. M. 1968, ApJ, 153, L59

O’Dwyer, B., Del Zanna, G., Mason, H. E., Weber, M. A., \& Tripathi, D. 2010, A\&A, 521, A21

Siarkowski, M., Falewicz, R., \& Rudawy, P. 2009, ApJ, 705, L143

Silva, A. V. R., Wang, H., Gary, D. E., Nitta, N., \& Zirin, H. 1997, ApJ, 481, 978

Smith, D. M., Lin, R. P., Turin, P., et al. 2002, Sol. Phys., 210, 33

Wang, T. J., Brosius, J. W., Thomas, R. J., Rabin, D. M., \& Davila, J. M. 2010, ApJS, 186, 222

Wang, T. J., Thomas, R. J., Brosius, J. W., et al. 2011, ApJS, 197, 32

Zarro, D. M., Canfield, R. C., Strong, K. T., \& Metcalf, T. R. 1988, ApJ, 324, 582 